\title{
Manejo bimodal de aneurismas asociados a malformaciones arteriovenosas cerebrales. Reporte de caso y breve revisión de la literatura
}

\author{
Bimodal management of aneurysms associated with cerebral arteriovenous \\ malformations. Case report and brief review of the literature
}

\author{
Carlos F. Nicolás-Cruz ${ }^{*}$, Michel G. Mondragón-Soto², José R. Aguilar Calderón ${ }^{3}$ y
}

Gustavo Melo-Guzmán ${ }^{4}$

${ }^{1}$ Servicio de Cirugía General, Instituto Mexicano del Seguro Social (IMSS); ${ }^{2}$ Servicio de Neurocirugía, Instituto Nacional de Neurología y Neurocirugía Manuel Velasco Suarez; ${ }^{3}$ Servicio de Neurocirugía, Hospital Central Sur de Alta Especialidad, Petróleos Mexicanos (PEMEX); ${ }^{4}$ Unidad de Terapia Endovascular Neurológica, Servicio de Neurocirugía, Hospital Juárez de México, Secretaría de Salud. Ciudad de México, México

\begin{abstract}
Resumen
La patogénesis de los aneurismas intracraneales asociados a malformaciones arteriovenosas cerebrales no es bien entendida y es aún objeto de discusión. Las decisiones sobre cuándo y cómo tratar los aneurismas intracraneales de estas características siempre han sido un reto terapéutico tanto para neurocirujanos vasculares como para terapistas endovasculares neurológicos. Reportamos el caso de una paciente de 51 años con aneurismas múltiples asociados a una malformación arteriovenosa, así como su manejo neuroquirúrgico, con un análisis comparativo con lo publicado en la literatura médica y científica en los últimos 10 años.
\end{abstract}

Palabras clave: Aneurisma cerebral. Clipaje. Embolización. Malformación arteriovenosa cerebral.

\begin{abstract}
The pathogenesis of intracranial aneurysms associated with arteriovenous malformations is not well understood and is still under discussion; the decisions about when and how to treat intracranial aneurysms of these characteristics have always been a therapeutic challenge for both, vascular neurosurgeons and endovascular neurological therapists. We report the case of a 51-year-old patient with multiple aneurysms associated with arteriovenous malformation, as well as her neurosurgical management, with a comparative analysis what has been published in the medical and scientific literature in the last 10 years.
\end{abstract}

Key words: Cerebral aneurysm. Clipping. Embolization. Cerebral arteriovenous malformation.

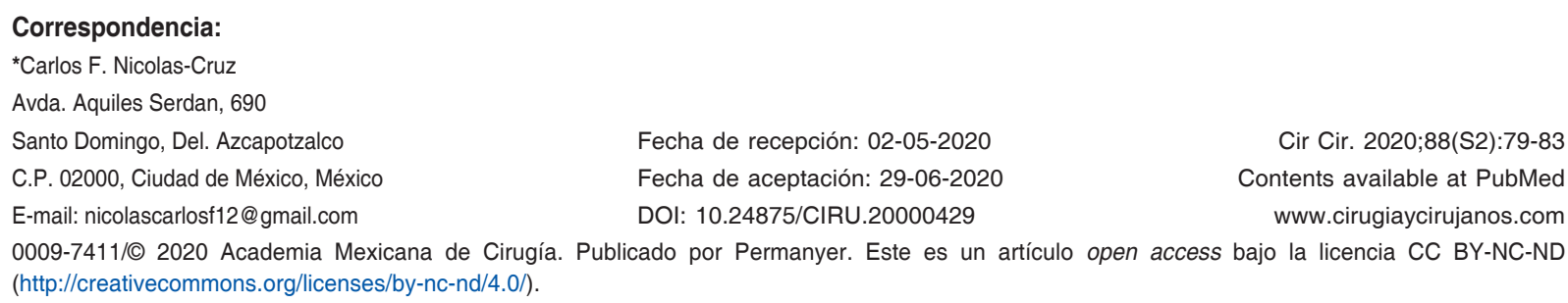




\section{Introducción}

Las malformaciones arteriovenosas cerebrales son anormalidades congénitas en los vasos sanguíneos derivadas de alteraciones en el desarrollo de la red capilar que permiten conexiones directas entre las arterias y las venas cerebrales ${ }^{1}$. Además, muestran evidencia de angiogénesis activa, respuesta inflamatoria y cambios estructurales, como interrupción o duplicación de la lámina elástica interna, convirtiéndolas en una derivación arteriovenosa de alto flujo con dilatación en sus arterias de alimentación y arterialización de las venas de drena$\mathrm{je}^{2}$. Su incidencia y su prevalencia aún no se conocen con certeza, pero algunos estudios han estimado que es de unas 15 por 100,000 personas $^{3}$, y tienen un riesgo de hemorragia de aproximadamente el $1-4 \%$ anual, dependiendo de la localización, el drenaje o el antecedente de hemorragia. A esto se añade la presencia de aneurismas intracraneales asociados, estimándose una prevalencia del $5-30 \%{ }^{4}$, que incrementa el riesgo de presentación hemorrágica del $43 \%$ al $61 \%{ }^{5}$. La presentación clínica con que se manifiesta más comúnmente es la hemorragia cerebral, en aproximadamente un 50\% de los casos; sin embargo, también son frecuentes convulsiones, cefalea y déficit neurológico focal ${ }^{6}$. Para diagnosticar con certeza que se trata de una malformación arteriovenosa $o$ un aneurisma, la angiografía con sustracción digital se ha convertido en el método de referencia, ya que su capacidad de detección es aún mayor que la de la tomografía computarizada y la angiografía por resonancia magnética, llegando a detectar aneurismas incluso menores de $3 \mathrm{~mm}$ de diámetro; sin embargo, no está exenta de riesgos $(<0.1 \%)$, entre los que destacan la hemorragia intracraneal, la lesión renal y la exposición a radiación?.

La carencia de comprensión de la relación entre los aneurismas y las malformaciones arteriovenosas ha llevado a la implementación de estrategias de tratamiento variadas, como son la resección quirúrgica o el clipaje transcraneal, la radiocirugía estereotáctica y la embolización endovascular. No obstante, el verdadero desafío gira en torno a cómo tratar las lesiones, en qué orden y en qué momento ${ }^{8}$.

Se han propuesto varias clasificaciones para los aneurismas asociados a malformaciones arteriovenosas, que pueden ser divididos grosso modo en prenidales, intranidales y posnidales. Los primeros son exclusivamente arteriales y pueden subdividirse en aneurismas no relacionados con el flujo, relacionados distalmente con el flujo y proximales al flujo?.

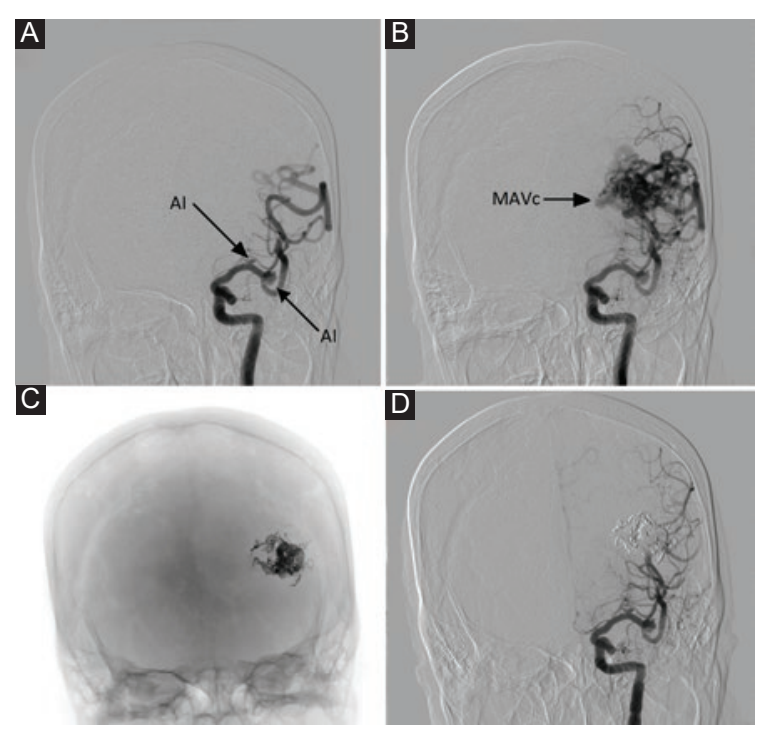

Figura 1. A: angiografía con sustracción digital en proyección anteroposterior en la que se observa un aneurisma pequeño del segmento M1 y un aneurisma mediano de la bifurcación de la arteria cerebral media izquierda. B: malformación arteriovenosa parietooccipital izquierda de $23 \times 26 \times 22 \mathrm{~mm}$ con drenaje profundo, Spetzler-Martin II. C: molde de ónix de la malformación arteriovenosa embolizada. D: control angiográfico tras la embolización.

En el presente trabajo describimos el caso y el manejo terapéutico neuroquirúrgico de una paciente con una malformación arteriovenosa occipitoparietal izquierda y presencia de aneurismas múltiples asociados en una de sus arterias aferentes, y realizamos una revisión de la literatura indexada y publicada en los últimos 10 años en revistas de alto y mediano impacto, para lo cual se realizó una revisión exhaustiva en diversas plataformas y bases de datos, tales como PubMed, NCBI, Medline y Ovid.

\section{Caso clínico}

Mujer de 51 años, sin antecedentes médicos ni quirúrgicos, con historia de cefalea de 30 años de evolución que se presentaba de tres a cinco veces por mes, de tipo holocraneana, pulsátil, de moderada intensidad, que cedía con la ingesta de antiinflamatorios no esteroideos, sin alteraciones neurológicas asociadas. Incidentalmente, por el estudio de una masa en el cuello que se protocolizó con estudios de imagen, se evidenció un cortocircuito arteriovenoso parietoccipital medial izquierdo, por lo que es referida al servicio de neurocirugía, donde se le realiza angiografía diagnóstica y se encuentra una malformación arteriovenosa de $33 \times 26 \times 32 \mathrm{~mm}$ en la región parietooccipital izquierda, que recibía suministro sanguíneo a través de las 


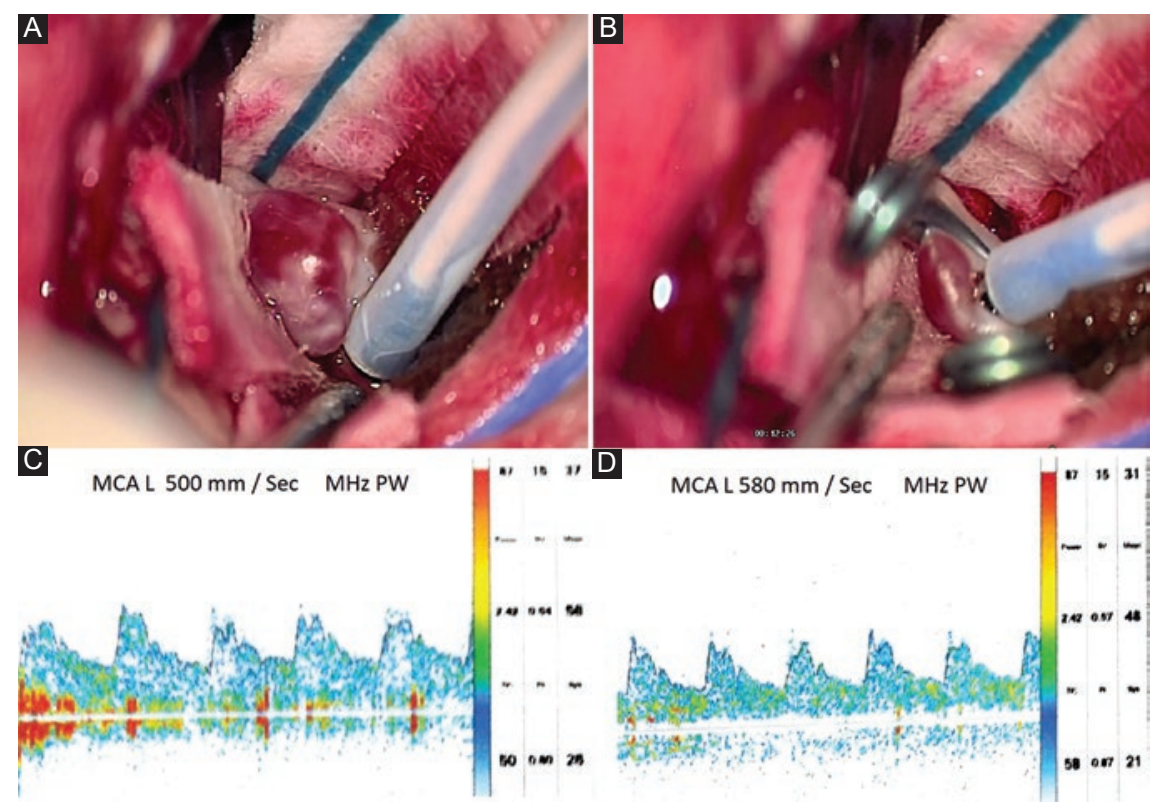

Figura 2. A-B: fotografías del clipaje del aneurisma de mayor tamaño en la bifurcación de la arteria cerebral media izquierda a través de una ventana pterional con monitorización Doppler. C-D: controles de velocidades de flujo antes y después del clipaje del aneurisma con Doppler transcraneal.
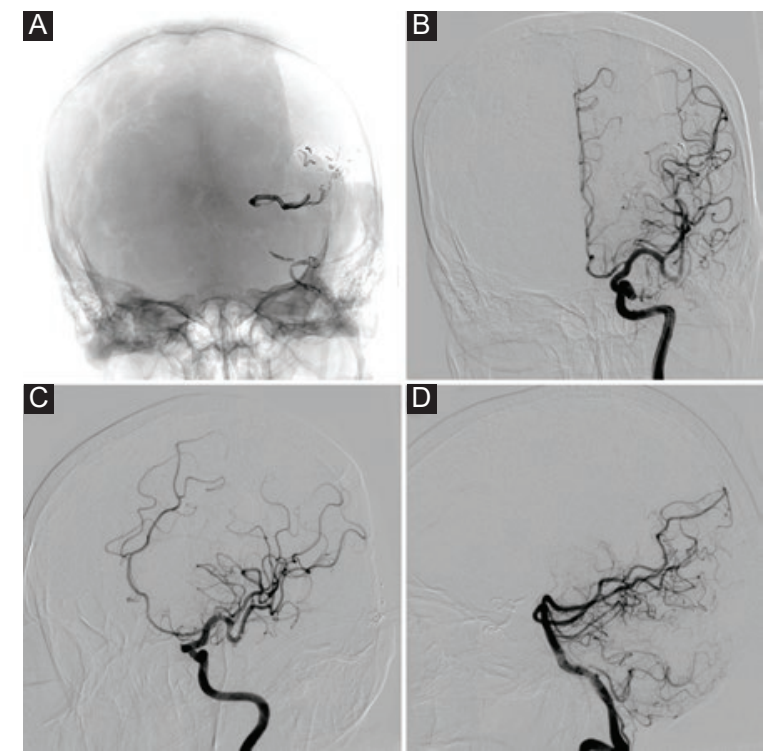

Figura 3. A: control angiográfico a los 6 meses del clipaje de los aneurismas de arteria cerebral media izquierda y tras la resección quirúrgica de la malformación arteriovenosa parietooccipital izquierda Spetzler-Martin II. B: angiografía con sustracción digital y proyección anteroposterior. C: angiografía con sustracción digital y proyección oblicua. D: angiografía con sustracción digital y proyección lateral.

arterias cerebral media izquierda y cerebral posterior homolateral, con drenaje venoso superficial hacia el seno sagital superior y el seno transverso, clasificándose como de grado III en la escala de Spetzler-Martin, con aneurismas proximales de $3.1 \times 2 \times 3.3 \mathrm{~mm}$ en el segmento M1 y de $5.7 \times 6.2 \times 7 \mathrm{~mm}$ en la bifurcación de la arteria cerebral media izquierda. Se plantea como manejo inicial la embolización de los aneurismas proximales con material cohesivo, pero durante la intervención endovascular se observa una compresión de la arteria temporal posterior, por lo cual se decide retirar el material cohesivo y dar por concluido el procedimiento de manera incompleta. Posteriormente se plantea embolizar la malformación arteriovenosa a través de la arteria occipital izquierda, logrando una embolización del 90\% (Fig. 1), y en un segundo tiempo quirúrgico se realiza el clipaje del aneurisma de mayor tamaño, a través de un abordaje pterional izquierdo, monitorizando con Doppler transcraneal las velocidades de flujo sanguíneo durante todo el procedimiento (Fig. 2). Finalmente se concluye sin complicaciones y la evolución en su recuperación es adecuada. A los 6 meses se realiza una craneotomía para la resección completa de las malformaciones arteriovenosas cerebrales, que concluye sin complicaciones, con sangrado mínimo y adecuada recuperación neurológica (Fig. 3).

\section{Discusión}

Existe una amplia variación en la literatura sobre la prevalencia de los aneurismas asociados a 
malformaciones arteriovenosas cerebrales, que va del 2.7 al $58 \%$, siendo el tipo relacionado con el flujo el más frecuentemente encontrado, hasta en el $78 \%$ de las malformaciones de gran tamaño, sobre todo de los tipos III y IV. El riesgo de sangrado es mayor cuando estos se encuentran infratentoriales ${ }^{10}$.

Se han aplicado diferentes sistemas de clasificación en un intento de comprender mejor la evolución natural de esta combinación de lesiones y las implicaciones para el tratamiento. El sistema de clasificación mejor aceptado es el propuesto por Redekop, et al., ${ }^{11}$ ya que toma en cuenta factores que influyen en la formación de aneurismas, como son el aumento del flujo sanguíneo regional, la disminución de las resistencias y el aumento de la turbulencia hemodinámica en los vasos alimentadores ${ }^{9}$. Sin embargo, cuando el cuadro se inicia con hemorragia intracraneal es difícil decidir a qué atribuir la rotura, sobre todo en aneurismas de flujo relacionado ${ }^{12}$. Diversos autores sugieren que cuanto más cercano esté el aneurisma a la malformación más probable es que sea este el causante de la hemorragia, y que los aneurismas de flujo no relacionado deberían ser tratados como una patología diferente y clasificarse como un hallazgo incidental ${ }^{13}$. Partiendo de esta premisa, en el caso reportado se decidió como manejo inicial la embolización del aneurisma de mayor tamaño, ya que este se encontraba en el punto de corte anatómico para considerarse de flujo relacionado.

La preocupación de que la eliminación abrupta de una malformación arteriovenosa cerebral pueda poner a un aneurisma ubicado a lo largo de las arterias de alimentación en riesgo inmediato de distensión y rotura ha llevado a algunos neurocirujanos a recomendar el tratamiento del aneurisma antes que el de la malformación ${ }^{14}$. Sin embargo, otros proponen eliminar primero la malformación arteriovenosa, ya que la alteración hemodinámica resultante de la reducción del flujo a través de las arterias de alimentación puede conducir a la disminución o a la regresión completa de los aneurismas relacionados hasta en un $14 \%$ de los casos $^{15}$. Por ello, en nuestro caso, al concluir de manera incompleta la embolización del aneurisma, decidimos de manera expedita realizar la embolización de la malformación arteriovenosa.

En la actualidad, el N-butil cianoacrilato (NBCA), el copolímero de etilen-vinil-alcohol vinílico (EVOH) y, en menor medida, los coils de platino y las partículas de polialcohol vinílico, se utilizan para el tratamiento endovascular. En la malformación arteriovenosa presentada se decidió embolizar con $\mathrm{EVOH}$, ya que es más manejable en comparación con NBCA y solidifica más lentamente, reduciendo el riesgo de atrapamiento del microcatéter; además, es posible realizar inyecciones prolongadas y repetidas dentro del mismo pedículo, permitiendo su llegada más distalmente hacia y dentro del nido ${ }^{16}$.

Los objetivos finales del tratamiento de las malformaciones arteriovenosas cerebrales son prevenir la hemorragia, evitar el deterioro neurológico que esta patología implicaría y eliminar el riesgo de mortalidad asociado con eventos hemorrágicos recurrentes. Sin embargo, el tratamiento solo es justificable si los riesgos relacionados con una intervención son menores o equivalentes a los riesgos a largo plazo de discapacidad o de muerte causados por la lesión misma. En caso de intervenir, se deberá documentar por angiografía posoperatoria la obliteración o extirpación total ${ }^{17}$.

En el caso específico de malformaciones arteriovenosas cerebrales asociadas con aneurismas intracraneales, se deben tener en cuenta varios factores antes de recomendar el tratamiento de una o ambas lesiones. Actualmente no hay consenso en la literatura sobre las mejores estrategias de manejo. Por ello decidimos, tras la embolización de la malformación arteriovenosa cerebral, clipar en un segundo tiempo el aneurisma embolizado de manera incompleta; en cambio, el aneurisma de menor tamaño, caracterizado por no estar relacionado con el flujo, se redujo por completo después de la embolización de la malformación arteriovenosa.

La utilización del Doppler transcraneal en el clipaje de aneurismas tiene el beneficio de ser no invasivo y de monitorizar en tiempo real los cambios hemodinámicos, previniendo y permitiendo tratar de manera oportuna el vasoespasmo cerebral cuando se llega a presentar. Tiene una sensibilidad del $83 \%$ y una especificidad del $88 \%$, y es de mayor utilidad en el clipaje de aneurismas que en su tratamiento endovascular. Se ha descrito que velocidades de flujo de 120-140 $\mathrm{cm} / \mathrm{s}, 140-200 \mathrm{~cm} / \mathrm{s}$ y $>200 \mathrm{~cm} / \mathrm{s}$ en la arteria cerebral media $^{18}$, que es donde se ubicaba el transductor utilizado en nuestro caso, son concordantes con vasoespasmo leve, moderado y grave, respectivamente. Durante nuestro procedimiento se mantuvo en 50-58 $\mathrm{cm} / \mathrm{s}$ antes, durante y posterior al clipaje.

\section{Conclusión}

Las malformaciones arteriovenosas cerebrales son unas de las lesiones más interesantes y desafiantes 
tratadas por el cirujano cerebrovascular, debido a que implica poner en práctica la habilidad más importante para cualquier médico: el juicio clínico. A esto se añade la presencia de aneurismas concomitantes a la lesión, que hace aún más difícil esta tarea. Actualmente no hay consenso sobre cuándo, cómo y en qué orden tratarlas, por lo que se deben tomar en consideración diversos factores para obtener resultados satisfactorios. En nuestra experiencia, la embolización inicial de la malformación arteriovenosa cerebral previo al clipaje de un aneurisma que se encuentre en el límite para ser considerado de flujo relacionado es segura, y la monitorización con Doppler transcraneal ha mostrado ser de gran utilidad para la prevención del vasoespasmo en el momento de la intervención quirúrgica.

\section{Conflicto de intereses}

Todos los autores involucrados en la concepción de la idea, la estructuración y la publicación de este manuscrito declaran no tener ningún conflicto de intereses.

\section{Responsabilidades éticas}

Protección de personas y animales. Los autores declaran que los procedimientos seguidos se conformaron a las normas éticas del comité de experimentación humana responsable y de acuerdo con la Asociación Médica Mundial y la Declaración de Helsinki.

Confidencialidad de los datos. Los autores declaran que han seguido los protocolos de su centro de trabajo sobre la publicación de datos de pacientes.

Derecho a la privacidad y consentimiento informado. Los autores han obtenido el consentimiento informado de los pacientes y/o sujetos referidos en el artículo. Este documento obra en poder del autor de correspondencia.

\section{Bibliografía}

1. Salomon R, Connolly E. Arteriovenous malformation of the brain. N Engl J Med. 2017;376:1859-66.

2. Kremer P, Koeleman P, Pawlikowska L, Weinsheimer S, Bendjilali N, Sidney $\mathrm{S}$, et al. Evaluation of genetic risk loci for intracranial aneurysms in sporadic arteriovenous malformation of the brain. J Neurol Neurosurg Psychiatry. 2015;86:524-9.

3. Nikolaev S, Vetiska S, Bonilla X, Bourdreau E, S Jauhuainen, Rezai Jahromi B, et al. Somatic activating KRAS mutations in arteriovenous malformations of the brain. N Engl J Med. 2018;378:250-6.

4. Morgan MK, Alsahli K, Wiedmann M, Assaad NN, Heller GZ. Factors associated with proximal intracranial aneurysms to brain arteriovenous malformations: a prospective cohort study. Neurosurgery. 2016;78:787-92.

5. Platz J, Berkefeld J, Singer OC, Wolff R, Seifert V, Konczalla J, et al. Frequency, risk of hemorrhage and treatment considerations for cerebral arteriovenous malformations with associated aneurysms. Acta Neurochir. 2014;156:2025-34.

6. Lv X, Li Y, Yang X, Jiang C, Wu Z. Characteristics of arteriovenous malformations associated with cerebral aneurysms. World Neurosurg. 2011;76:288-91.

7. Derdeyn CP, Zipfel GJ, Albuquerque FC, Cooke DL, Feldmann E, Sheehan JP, et al. Management of brain arteriovenous malformations: a scientific statement for healthcare professionals from the American Heart Association/American Stroke Association. Stroke. 2017;48:e200-24.

8. Elhammady MS, Aziz-Sultan MA, Heros RC. The management of cerebral arteriovenous malformations associated with aneurysms. World Neurosurg. 2013;80:e123-9.

9. D'Aliberti G, Talamonti G, Cenzato M, La Camera A, Debernardi A, Valvassori $L$, et al. Arterial and venous aneurysms associated with arteriovenous malformations. World Neurosurg. 2015;83:188-96.

10. Cagnazzo F, Brinjikji W, Lanzino G. Arterial aneurysms associated with arteriovenous malformations of the brain: classification, incidence, risk of hemorrhage, and treatment - a systematic review. Acta Neurochir. 2016;158:2095-104.

11. Redekop G, TerBrugge K, Montanera W, Willinsky R. Arterial aneurysms associated with cerebral arteriovenous malformations: classification, incidence, and risk of hemorrhage. J Neurosurg. 1998; 89(4):539-46.

12. Flores $B C$, Klinger DR, Rickert KL, Barnett SL, Welch BG, White JA, et al. Management of intracranial aneurysms associated with arteriovenous malformations. Neurosurg Focus. 2014;37:E11.

13. Lv X, Wu Z, Li Y, Yang X, Jiang C, Sun Y, et al. Endovascular treatment of cerebral aneurysms associated with arteriovenous malformations. Eur J Radiol. 2012;81:1296-8.

14. Kano H, Lunsford LD, Flickinger JC, Yang HC, Flannery TJ, Awan NR, et al. Stereotactic radiosurgery for arteriovenous malformations, Part 1 : management of Spetzler-Martin grade I and II arteriovenous malformations. J Neurosurg. 2012;116:11-20.

15. Signorelli F, Gory B, Pelissou-Guyotat I, Guyotat J, Riva R, Dailler $F$, et al. Ruptured brain arteriovenous malformations associated with aneurysms: safety and efficacy of selective embolization in the acute phase of hemorrhage. Neuroradiology. 2014;56:763-9.

16. Ellis JA, Lavine SD. Role of embolization for cerebral arteriovenous malformations. Methodist Debakey Cardiovasc J. 2014;10:234-9.

17. Mohr JP, Parides MK, Stapf C, Moquete E, Moy CS, Overbey JR, et al. Medical management with or without interventional therapy for unruptured brain arteriovenous malformations (ARUBA): a multicentre, non-blinded, randomised trial. Lancet. 2014;383:614-21.

18. Wang $Y$, Ma Y, Hui P, Liu G, Luan Y, Wang C. Evaluation of application value of transcranial Doppler (TCD) in the inspection of cerebral vasospasm after the treatment of intracranial aneurysm. Curr Med Imaging Rev. 2018;14:143-6. 